\title{
Elevated Concentrations of Neurofilament Light Chain in the Cerebrospinal Fluid of Bipolar Disorder Patients
}

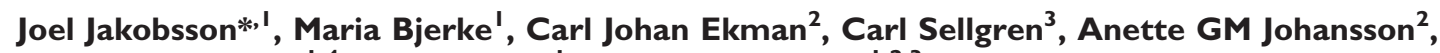 \\ Henrik Zetterberg ${ }^{1,4}$, Kaj Blennow' and Mikael Landén ${ }^{1,2,3}$ \\ 'Institute of Neuroscience and Physiology, Department of Psychiatry and Neurochemistry, Sahlgrenska Academy, University of Gothenburg, \\ Gothenburg, Sweden; 'Department of Clinical Neuroscience, Karolinska Institutet, Stockholm, Sweden; ${ }^{3}$ Department of Medical Epidemiology \\ and Biostatistics, Karolinska Institutet, Stockholm, Sweden; ${ }^{4}$ UCL Institute of Neurology, London, UK
}

\begin{abstract}
Bipolar disorder (BD) is characterized by mood swings between manic and depressive states. The etiology and pathogenesis of BD is unclear, but many of the affected cognitive domains, as well as neuroanatomical abnormalities, resemble symptoms and signs of small vessel disease. In small vessel disease, cerebrospinal fluid (CSF) markers reflecting damages in different cell types and subcellular structures of the brain have been established. Hence, we hypothesized that CSF markers related to small vessel disease may also be applicable as biomarkers for BD. To investigate this hypothesis, we sampled CSF from 133 patients with BD and 86 healthy controls. The concentrations of neurofilament light chain (NF-L), myelin basic protein (MBP), SIOOB, and heart-type fatty acid binding protein (H-FABP) were measured in CSF and analyzed in relation to diagnosis, clinical characteristics, and ongoing medications. Hereby we found an elevation of the marker of subcortical axonal damage, NF-L, in bipolar subjects. We also identified positive associations between NF-L and treatment with atypical antipsychotics, MBP and lamotrigine, and $\mathrm{H}-\mathrm{FABP}$ and lithium. These findings indicate axonal damage as an underlying neuropathological component of bipolar disorder, although the clinical value of elevated NF-L remains to be validated in follow-up studies. The associations between current medications and CSF brain injury markers might aid in the understanding of both therapeutic and adverse effects of these drugs.
\end{abstract}

Neuropsychopharmacology (2014) 39, 2349-2356; doi:I0.1038/npp.2014.8I; published online 23 April 2014

\section{INTRODUCTION}

Bipolar disorder (BD) is a chronic mood disorder characterized by mood swings between manic and depressive states (Belmaker, 2004). BD affects $\sim 1-3 \%$ of the population (Merikangas et al, 2011) and entails high costs for the society (Ekman et al, 2013) and is associated with personal suffering, functional impairment, premature mortality, and higher risk for other psychiatric and medical disorders (Kupfer, 2005). Because of the high recurrence risk, prophylactic maintenance therapy is the cornerstone of long-term management of BD. The first-line mood-stabilizing treatments include lithium, divalproex, olanzapine, and lamotrigine (the latter mainly for those with mild manias) (Yatham et al, 2009). BD has also been coupled to cognitive impairment including reduced abilities in executive function and verbal memory (Martinez-Aran et al, 2000; Zarate et al, 2000; Martinez-Aran et al, 2005; Robinson et al, 2006; Sanchez-Moreno et al, 2009; Palsson et al, 2013). Structural

\footnotetext{
*Correspondence: Dr J Jakobsson, Sahlgrenska University Hospital, Sahlgrenska Academy, University of Gothenburg, Blå Stråket 15, Floor 3, Gothenburg SE-4I3 45, Sweden, Tel: +46 31342 4522, Fax: +46 31342 1533, E-mail: joel.jakobsson@neuro.gu.se Received 2 October 2013; revised 7 February 2014; accepted 17 March 2014; accepted article preview online 3 April 2014
}

imaging studies have suggested that the decline in cognitive function is associated with morphological abnormalities of the brain (Altshuler et al, 1995; McDonald et al, 2004; Kempton et al, 2008; Konarski et al, 2008), and that the number of manic episodes is associated with decreased gray matter in dorsolateral prefrontal cortex (Ekman et al, 2010). The most frequent abnormal morphological findings are lateral ventricular enlargements and increased rates of deep white matter hyperintensities (Kempton et al, 2008; Beyer et al, 2009). The association between white matter pathology and $\mathrm{BD}$ has been further supported by morphometric studies showing reductions in total white matter volumes (Strakowski et al, 1993; Kieseppa et al, 2003; Davis et al, 2004; Rosso et al, 2007). Patients with cerebral small vessel disease share common features with bipolar patients such as poor performance on tests of executive function and processing speed, generalized brain atrophy as well as ventricular enlargement, blood brain barrier (BBB) dysfunction, and white matter changes in periventricular as well as in the deep white matter (Chui, 2007). In small vessel disease, the periventricular lesions in particular have been associated with cognitive impairment and a decline in executive function and processing speed (Bolandzadeh $\mathrm{et} \mathrm{al}$, 2012), whereas deep white matter changes have been associated with depressive symptoms (Krishnan et al, 2006). The effects of cerebral small vessel disease have 
been characterized at the biochemical level by analyses of cerebrospinal fluid (CSF). Products found in CSF are thought to reflect some aspects of brain biochemistry and markers of brain injury may thus be established (Bjerke et al, 2011). CSF markers have the potential to act as diagnostics tools but can also function as predictors for treatment response, side effects, and/or disease progression (Perlis, 2011). In a previous study, we investigated a potential Alzheimer-like component of BD by analyzing established CSF markers for neurodegenerative disorders (T-tau, P-tau, and amyloid $\beta$ ), but observed no major differences between patients and controls (Jakobsson et al, 2013). There are, however, other CSF markers that reflect other aspects of brain injuries such as white matter lesions and associated glial cell injury as well as neurodegenerative disease. These biomarkers include neurofilament light chain (NF-L), a marker of subcortical myelinated axons (Rosengren et al, 1996); myelin basic protein (MBP), a marker of oligodendrocytes (Olsson et al, 2011); the calcium-binding protein S100B, which is enriched in and might be secreted by astro- and oligodendrocytes (Schroeter et al, 2013); and heart-type fatty acid binding protein (H-FABP), which despite its name is a marker for cortical neurons (Pelsers et al, 2004).

Based on the cognitive and morphological similarities between $\mathrm{BD}$ and cerebral small vessel disease, we hypothesized that CSF markers reflecting brain injury are altered in $\mathrm{BD}$. To investigate this, we assessed the CSF concentrations of NF-L, MBP, S100B, and H-FABP in patients with BD and healthy controls. We also evaluated the results in relation to clinical characteristics and ongoing medication.

\section{SUBJECTS AND METHODS}

\section{Study Population}

Patients were recruited from the St Göran bipolar project, enrolling patients from the bipolar unit at the Northern Stockholm Psychiatric Clinic, Stockholm, Sweden. The work-up and diagnostic assessments have been described in detail previously (Ryden et al, 2009; Jakobsson et al, 2013). The general criteria for inclusion were patients at least 18 years old and who met the Diagnostic and Statistical Manual of mental disorders (DSM)-IV criteria for BD type I, type II, cyclothymia, or not otherwise specified (NOS). Information was collected about age, gender, education, living condition, primary source of income, total number of episodes, duration of illness (defined as age at sampling minus age at first manic/hypomanic episode), body mass index (BMI), and history of psychosis. The severity of $\mathrm{BD}$ was rated using the Clinical Global Impression (CGI) rating scale and Global Assessment of Functioning (GAF). The CGI scale reflects the lifetime severity of $\mathrm{BD}$ using a 7-point scale: $1=$ normal or not at all ill, $2=$ borderline mentally ill, $3=$ mildly ill, $4=$ moderately ill, $5=$ markedly ill, $6=$ severely ill, and $7=$ extremely ill. GAF is a 100 -point scale used by clinicians to rate the general functional capacity of patients in a social, occupational, and psychological perspective $(100=$ superior functioning and $1=$ minimal functioning). A split version of GAF was used in this study, ie, GAF function (GAF-F) and GAF symptom (GAF-S). For ethical reasons, patients continued to take their prescribed medications at the time of CSF sampling. Age- and sexmatched healthy, population-based controls were randomly selected by Statistics Sweden (SCB) and contacted by mail. The inclusion criteria for the healthy controls have been described previously (Jakobsson et al, 2013). The study was approved by the Regional Ethics Committee in Stockholm and conducted in accordance with the latest Helsinki Protocol. After complete description of the study, all enrolled patients and controls consented orally and in writing to participate in the study.

\section{CSF Sampling}

CSF sampling (lumbar puncture) was performed when the participants were in a stable euthymic mood. Subjects fasted overnight before the CSF collection that occurred between 0900 and $1000 \mathrm{~h}$. The spinal needle was inserted into the L3/L4 or L4/L5 interspace and a total volume of $12 \mathrm{ml}$ of the CSF was collected, gently inverted to avoid gradient effects, and divided into $1.0-1.6 \mathrm{ml}$ aliquots that were stored at $-80^{\circ} \mathrm{C}$ pending analysis. An identical procedure was performed for the controls. All samples in this study were thawed and refrozen once before analysis. The staff performing the lumbar puncture was not blind with respect to whether the test person was a patient or control.

\section{Biomarker Analysis}

NF-L was analyzed as previously described with a commercial ELISA assay (NF-light, UmanDiagnostis AB, Umeå, Sweden). S100B was determined by an electrochemoluminescence immunoassay using the Modular system and the S100 reagent kit (Elecsys S100, Roche Diagnostics, Penzberg, Germany). MBP (MBP ELISA, Beckman Coulter, Brea, CA) and H-FABP (Human H-FABP, Hycult Biotechnology, Uden, The Netherlands) were measured using commercially available ELISA assays. All analyses were performed following the instructions from the manufacturer. Intra-assay coefficients of variation were $<10 \%$ for all assays. The laboratory technicians performing the analyses were blinded to patient identity.

\section{Statistics}

SPSS Statistics version 20 (IBM, Armonk, NY) was used in all statistical analyses. Data not showing normal distribution (as tested by one-sample Kolmogorov-Smirnov test) were log-transformed before parametric tests. Separate linear regressions (enter models) were used to analyze effects of age, gender, BMI, clinical characteristics, diagnosis, and current medications on CSF marker concentrations. All $p$-values are presented as two tailed and $p$-values of $<0.05$ were regarded as significant.

\section{RESULTS}

\section{Patient-Control Comparisons}

Demographics and clinical characteristics of the study population are presented in Table 1. Patients' current medication was classified as use or nonuse of each medication (Table 1). Many patients were, however, on a combination 
Markers of brain injury in bipolar disorder

J Jakobsson et al

Table I Demographics and Clinical Characteristics of the Study Population

\begin{tabular}{|c|c|c|c|c|}
\hline \multirow[t]{2}{*}{ Characteristic } & \multicolumn{2}{|c|}{ Healthy controls } & \multicolumn{2}{|c|}{ Bipolar disorder } \\
\hline & $\mathbf{N}$ & $\%$ & $\mathbf{N}$ & $\%$ \\
\hline
\end{tabular}

Education

Not completed compulsory school

Completed compulsory school

0

$0.0-3$

2.3

Completed upper secondary school

Completed higher education $<3$ years

39.5

25.6

Completed higher education $>3$ years

Living condition $^{\mathrm{a}}$

\section{Alone}

With partner

With parent/parents

With other adults

Primary source of income

Employment/education

Sickness benefit

Supplementary benefit

Relative's or own capital

\section{Diagnosis}

Bipolar disorder type I

Prior psychosis

$\begin{array}{ll}65 & 48.9 \\ 46 & 34.6 \\ 22 & 16.5 \\ 64 & 50\end{array}$

Medications

Lithium

\begin{tabular}{ll}
78 & 58.6 \\
30 & 22.6 \\
16 & 12.0 \\
62 & 46.6 \\
27 & 20.3 \\
29 & 21.8 \\
\hline
\end{tabular}

Lamotrigine

Divalproex

Antidepressants

Anxiolytics

Atypical APs

\section{Median}

IQR

Median

Age (years)

BMI

Number of episodes

Duration of illness

GAF-F

GAF-S

CGI

YMRS

MADRS

28-46

21.6-25.7

23.4

35

24.7

10

11

68

68

4

28-50

22.2-27.7

6-20

4-20

60-72

60-75

4-5

$0-1$

0-4

Abbreviations: APs, antipsychotics; IQR, interquartile range; BMI, body mass index; GAF, Global Assessment of Functioning ( $S=$ symptom and F= function); CGI, Clinical Global Impression; YMRS, Young Mania Rating Scale; MADRS, Montgomery-Åsberg Depression Rating Scale.

${ }^{a}$ Data missing in four cases.

bipolar disorder spectrum diagnoses other than type I or type II. 

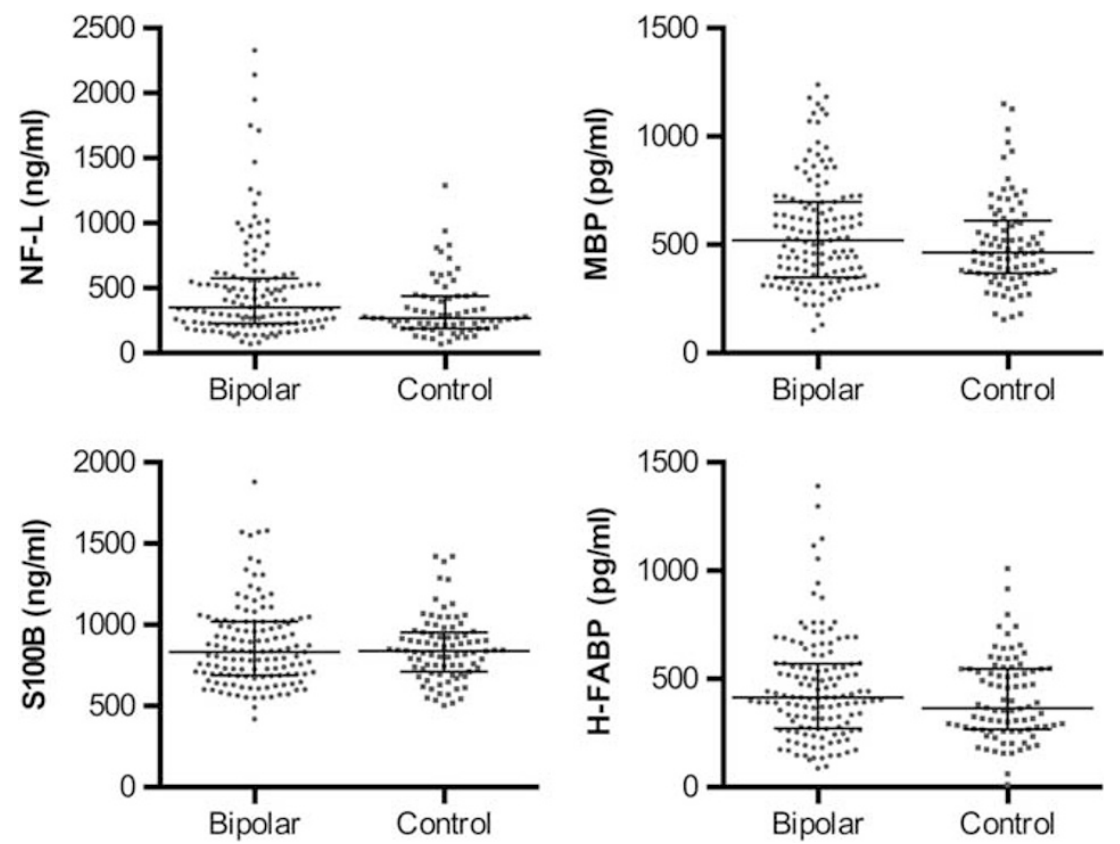

Figure I Grouped scatter plot showing the cerebrospinal fluid concentrations of neurofilament light chain (NF-L), myelin basic protein (MBP), the calcium-binding protein SIOOB (SIOOB), and heart-type fatty acid binding protein (H-FABP). The median is shown as a straight line and the interquartile range is shown as error bars.

Table 2 Comparisons of CSF Biomarker Concentrations Between Bipolar Disorder Patients and Healthy Controls (Linear Regression with Age and Gender as Covariates)

\begin{tabular}{|c|c|c|c|c|c|c|c|c|}
\hline \multirow[t]{2}{*}{ Biomarker } & \multicolumn{2}{|c|}{ Controls $\left(N=86^{a}\right)$} & \multicolumn{2}{|c|}{ Bipolar disorder $(N=133)$} & \multicolumn{4}{|c|}{ Analysis } \\
\hline & Mean $^{b}$ & SE & Mean $^{b}$ & SE & $\beta$ & $t$ & d.f. & $P$ \\
\hline MBP (pg/ml) & 508 & 19 & 553 & 15 & 0.094 & 1.835 & । & 0.068 \\
\hline SIOOB (pg/ml) & 850 & 23 & 873 & 19 & 0.047 & 0.754 & । & 0.452 \\
\hline
\end{tabular}

Abbreviations: NF-L, neurofilament light chain; MBP, myelin basic protein; SI00B, calcium-binding protein SI 00B; h-FABP, heart-type fatty acid binding protein. ${ }^{a} N=7 \mid$ for NF-L.

${ }^{b}$ Estimated marginal means adjusted for age and gender (H-FABP: only adjusted for age).

'Log transformed before statistical analysis.

of medications and no patients were untreated at the time of CSF sampling. CSF concentrations of NF-L, MBP, S100B, and H-FABP in patients and controls are displayed in Figure 1. The concentrations of NF-L, MBP, and S100B correlated significantly with age (positive correlation) and gender (higher in males) in both patients and controls (Supplementary Table S1). H-FABP correlated significantly with age (positive correlation) and with gender (higher in males) at a trend level (Supplementary Table S1). None of the markers correlated with BMI. Thus, age and gender were used as covariates in further statistical analyses. The concentrations of NF-L were significantly higher in BD patients compared with controls $(p=0.001$, Table 2$)$. The concentrations of MBP, S100B, and H-FABP were not significantly different between patients and controls (Table 2).

\section{CSF Markers in Relation to Diagnosis and Clinical Characteristics}

We next tested whether alterations in CSF markers were associated with a certain type of bipolar diagnosis and/or variables reflecting disease intensity/severity, that is, history of episodes of psychosis, duration of illness, total number of episodes, CGI score, and GAF-S and GAF-F scores (Table 3). No correlations were observed between any of these variables and the CSF markers except for a negative association between NF-L and GAF-S-score as well as a 
Table 3 CSF Biomarker Concentrations in Relation to Clinical Variables (Separate Linear Regressions with Age and Gender as Covariates)

\begin{tabular}{|c|c|c|c|c|c|c|c|c|}
\hline & \multicolumn{2}{|c|}{ NF- $\mathbf{L}^{\mathrm{a}}$} & \multicolumn{2}{|c|}{ MBP } & \multicolumn{2}{|c|}{ SIOOB } & \multicolumn{2}{|c|}{ H-FABP } \\
\hline & $\beta$ & $P$ & $\beta$ & $P$ & $\beta$ & $P$ & $\beta$ & $P$ \\
\hline Type I $\left(\right.$ ref $\left.^{b}\right)$ & 0.000 & NA & 0.000 & NA & 0.000 & NA & 0.000 & NA \\
\hline Type ॥ & -0.025 & 0.706 & 0.141 & 0.052 & 0.046 & 0.595 & 0.010 & 0.908 \\
\hline Psychosis $^{c}$ & 0.085 & 0.343 & -0.106 & 0.239 & 0.022 & 0.804 & 0.114 & 0.205 \\
\hline GAF-F & -0.083 & 0.358 & -0.012 & 0.897 & -0.052 & 0.566 & 0.111 & 0.216 \\
\hline GAF-Sc & -0.162 & 0.070 & -0.044 & 0.624 & -0.069 & 0.445 & 0.034 & 0.706 \\
\hline CGI score ${ }^{d}$ & 0.046 & 0.609 & -0.087 & 0.336 & 0.056 & 0.532 & -0.073 & 0.417 \\
\hline Years of sickness ${ }^{e}$ & 0.066 & 0.473 & 0.072 & 0.432 & 0.054 & 0.553 & 0.149 & 0.102 \\
\hline
\end{tabular}

Abbreviations: NA, not applicable; NF-L, neurofilament light chain; MBP, myelin basic protein; SI00B, calcium-binding protein SI00B; h-FABP, heart-type fatty acid binding protein; CGl, Clinical Global Impression; GAF, Global Assessment of Function; NA, ....... .

aLog transformed before statistical analysis.

bipolar type I as reference group.

${ }^{\mathrm{C}}$ Data from 128 patients.

${ }^{\mathrm{d} D a t a}$ from 127 patients.

eData from 127 patients.

Table 4 CSF Biomarker Concentrations in Relation to Ongoing Medical Treatments (Separate Linear Regression with Age and Gender as Covariates)

\begin{tabular}{|c|c|c|c|c|c|c|c|c|}
\hline & \multicolumn{2}{|c|}{$N F-L^{a}$} & \multicolumn{2}{|c|}{ MBP } & \multicolumn{2}{|c|}{ SIOOB } & \multicolumn{2}{|c|}{ H-FABP } \\
\hline & $\beta$ & $P$ & $\beta$ & $P$ & $\beta$ & $P$ & $\beta$ & $P$ \\
\hline Divalproex & 0.046 & 0.599 & 0.094 & 0.283 & -0.075 & 0.395 & -0.143 & 0.103 \\
\hline Lamotrigine & 0.064 & 0.469 & 0.268 & 0.002 & 0.081 & 0.357 & 0.066 & 0.452 \\
\hline Atypical antipsychotics & 0.206 & 0.018 & 0.066 & 0.454 & 0.114 & 0.194 & 0.075 & 0.396 \\
\hline
\end{tabular}

Abbreviations: NF-L, neurofilament light chain; MBP, myelin basic protein; SI00B, calcium-binding protein SI00B; h-FABP, heart-type fatty acid binding protein.

${ }^{a}$ Log transformed before statistical analysis.

positive association between MBP and BDs type II at trend levels ( $p=0.070$ and $p=0.052$, respectively).

\section{CSF Markers in Relation to Medications}

The patients in this study continued to take their prescribed medications at the time of CSF sampling (Table 1). Thus, subgroups of patients were on different medications, making it possible to identify associations between CSF marker and medications. Three significant positive associations between CSF markers and ongoing medications were found: (1) atypical antipsychotics and NF-L ( $p=0.018) ;(2)$ lamotrigine and $\operatorname{MBP}(p=0.002)$; and (3) lithium and H-FABP $(p=0.043)$ (Table 4$)$. Notably, bipolar patients without atypical antipsychotics still differed significantly from controls regarding NF-L (Supplementary Table S2). With respect to $\mathrm{MBP}$ and $\mathrm{H}-\mathrm{FABP}$, patients without lamotrigine and lithium, respectively, did not differ from controls (Supplementary Table S2). No significant correlations were found between duration of atypical antipsychotic treatment and NF-L $(\beta=0.335, p=0.118, N=21$, linear regression with age and gender as covariates), duration of lamotrigine treatment and $\operatorname{MBP}(\beta=0.154, p=0.425$, $N=27$, linear regression with age and gender as covariates), or duration of lithium treatment and H-FABP $(\beta=0.195$, $p=0.097, N=71$, linear regression with age and gender as covariates). Furthermore, we investigated the clinical status of the different medication groups in this study population and found that compared with patients without treatment: (1) patients treated with antipsychotics had a lower GAF-F score $(p=0.030)$, higher CGI score $(p=0.006)$, and increased sick leave $(p=0.026)$ (Supplementary Table S3), (2) patients treated with lithium treatment were more frequently diagnosed with bipolar type I diagnosis $(p=0.035)$, lifetime 
episodes of psychosis $(p<0.001)$, and associated with a higher CGI score $(p=0.006)$ (Supplementary Table S4), and (3) patients treated with lamotrigine were more frequently treated with antidepressants $(p=0.037)$ and anxiolytics $(p=0.011)$ (Supplementary Table S5).

\section{DISCUSSION}

This is the first study of four established CSF markers that reflect brain injury in a large sample of $\mathrm{BD}$ patients and healthy controls. Patients with BD were found to have higher NF-L levels than healthy controls. The concentrations of the other markers (MBP, S100B, and H-FABP) were not significantly different between patients and controls.

Neurofilaments, of which NF-L is a subunit, are found in neurons and are particularly abundant in axons where they are major components of the axonal cytoskeleton (Perrot et al, 2008). Neuronal death or axonal degeneration will result in disintegration of the axonal membrane with a subsequent release of NF-L into the extracellular compartment followed by diffusion into the CSF. Thus, the CSF concentration of NF-L mirrors the degree of acute axonal degradation. Elevated concentrations of neurofilament subunits have been described in many neurological diseases including Alzheimer's disease (Sjogren et al, 2000; Brettschneider et al, 2006), vascular dementia (Bjerke et al, 2009), amyotrophic lateral sclerosis (Zetterberg et al, 2007; Lu et al, 2012), and multiple sclerosis (Salzer et al, 2010). In multiple sclerosis, the CSF concentration of NF-L is downregulated by treatment with the anti-inflammatory drug natalizumab, indirectly implicating that increased CSF NF-L reflects inflammatory-mediated axonal damage (Gunnarsson et al, 2011). Furthermore, high NF-L together with normal concentrations of the three core markers for Alzheimer's disease (ie, T-tau, P-tau, and amyloid $\beta$ ) has been shown to be a signature of dementia of the subcortical vascular type, that is, signifying small vessel disease accompanied by white matter changes (Bjerke et al, 2011). We previously analyzed T-tau, $\mathrm{P}$-tau, and $\mathrm{A} \beta 42$ in this study population and found normal concentrations of these markers (Jakobsson et al, 2013), strengthening the theory that pathology of small vessels may be implicated in bipolar disorder.

We found that ongoing treatment with atypical antipsychotics (eg, olanzapine and quetiapine) was associated with high NF-L concentrations. Interestingly, a longitudinal study of patients with schizophrenia suggested that treatment with antipsychotics is associated with decreased brain volume (Ho et al, 2011). In addition, treatment with atypical antipsychotics is known to be associated with a range of adverse effects on metabolic, neurologic, and/or cardiovascular systems (Cha and McIntyre, 2012). Even though the current cross-sectional study cannot address causality, it is conceivable that high NF-L in BD patients reflects adverse effects of atypical antipsychotic treatment. Notably, however, patients without atypical antipsychotics also had higher mean NF-L concentrations than controls and we found no significant correlation between NF-L and duration of atypical antipsychotic treatment. This speaks against an antipsychotic-induced brain injury and rather suggests that high NF-L is related to a certain subtype of bipolar patients that is more likely to be treated with atypical antipsychotics. This potential subtype of $\mathrm{BD}$ patients appeared to suffer from a more severe illness than patients without this treatment, as shown by lower GAF score and higher CGI score (although none of these variables had a significant direct effect on NF-L). There were, however, no associations between NF-L and subtypes of BD diagnosis, which suggests that NF-L is not a useful diagnostic marker of BD subtypes. It is possible that high NF-L is an indicator of brain injuries that will manifest later in life as cognitive impairments and/ or disease progression. Another hypothesis is that high NF$\mathrm{L}$ reflects subtle neurodevelopmental defects that may be a trait risk factor for $\mathrm{BD}$.

MBP is an important component of the myelin sheath and was early on proposed as a marker for multiple sclerosis as neuronal MBP concentrations rise in response to neuronal damage (Whitaker et al, 1980). Multiple sclerosis has long been associated with neuropsychiatric disorders where major depression is the most common comorbid disorder (eg, the major depression lifetime prevalence rate in MS patients is three times higher than in the general population) (Minden and Schiffer, 1990). Interestingly, elevated MBP concentrations were associated with patients with BD type II (trend level) that is more closely related to recurrent major depression. Thus, one could speculate that high MBP concentrations might be an indicator of depression-associated axonal damage similar to the degenerative processes observed in multiple sclerosis patients. We also observed an association between lamotrigine treatment and MBP. Indeed, patients with lamotrigine had significantly higher concentrations than controls, whereas patients without lamotrigine did not differ from controls. This finding accords findings from a clinical trial in which a neuroprotective effect of lamotrigine was tested in MS patients (Kapoor et al, 2010). Interestingly, the findings were negative and lamotrigine treatment was instead associated with white matter volume loss. Analogous to the interpretation of the NF-L and atypical antipsychotic association, it is possible that high MBP is related to a certain subtype of bipolar patients that is more likely to be treated with lamotrigine. In favor of this hypothesis, we found no significant association between duration of lamotrigine treatment and MBP.

Glial impairment has been suggested to be an underlying pathophysiological mechanism of BD (Rajkowska and Miguel-Hidalgo, 2007). The results of this study are not directly supporting this hypothesis as the astro- and oligodendrocytic marker S100B was unaltered. Several studies have, however, reported increased serum S100B concentrations during episodes of mania and depression (MachadoVieira et al, 2002; Schroeter et al, 2002; Andreazza et al, 2007; Schroeter et al, 2008). A study of serum from euthymic bipolar patients, however, reported no differences in $\mathrm{S100B}$, in line with the finding in the current study (Andreazza et al, 2007). It is possible that elevated CSF concentrations of S100B could have been detected in the current study if patients had been in a depressive or manic state. Thus, it cannot be excluded that S100B-related brain injuries and glial impairment are associated with the pathophysiology of BD. Biomarker concentrations in serum and CSF are, however, in many cases not correlated, and this can also explain the discrepancy between our study and 
previous studies. Importantly, S100B expression has been reported in several nonneuronal cells including adipocytes, melanocytes, chondrocytes, myocardium, and Schwann cells (reviewed by Schroeter et al, 2013). It is thus possible that manic and/or depressed episodes, as well as medications, affect S100B expression in these cells, resulting in increased S100B serum concentrations.

The CSF H-FABP concentrations were also unaltered in BD but we observed a slight elevation in lithium-treated patients. It is, however, unlikely that this elevation reflects neuronal loss as lithium treatment previously has been found to increase gray matter volume (Moore et al, 2000). In addition, we found no significant association between duration of lithium treatment and H-FABP. The lithiumtreated group in this study population represents mostly bipolar type I patients and patients with a history of psychotic episodes, although none of these variables were directly associated with H-FABP.

The cross-sectional design of the current study precludes causal inferences, which warrants longitudinal studies. Another limitation of this study is that many patients were on a combined treatment that might have influenced biomarker concentrations. In addition, no information about clinical status at treatment initiation or treatment history was available. This is the first study assessing these brain injury markers in BD and in order to avoid type II errors we chose not to adjust for multiple comparisons in the post hoc analyses. High NF-L in BD as well as the association between MBP and lamotrigine would, however, remain significant even after implementation of correction for multiple testing (ie, Bonferroni correction), whereas the other associations would not survive correction for multiple testing. Despite this, the associations may suggest important directions for future clinical and experimental studies, although verification of the current findings in an independent sample population is desirable before drawing any definite conclusions.

We conclude that the mean concentration of NF-L is elevated in $\mathrm{BD}$ patients as compared with healthy controls. This finding suggests axonal damage as an underlying neuropathological component of $\mathrm{BD}$. However, the usefulness of NF-L as a BD biomarker remains to be elucidated in follow-up studies. In addition, we conclude that MBP and H-FABP might have a potential for biomarkers to monitor adverse and/or therapeutic effects of lamotrigine and lithium, respectively.

\section{FUNDING AND DISCLOSURE}

The authors declare no conflict of interest.

\section{ACKNOWLEDGEMENTS}

We thank the staff at the St Görans Bipolar Affective Disorder unit, including coordinator Martina Wennberg, study nurses Agneta Carlswärd-Kjellin, Lena Lundberg, and Benita Gezelius, and data managers Haydeh Olofsson and Mathias Kardell, for the diagnostic assessments and enrolling patients for this study. Yngve Hallström and Auris Pelanis are acknowledged for performing lumbar punctures on patients and controls. Erik Joas and Kristoffer Bäckman are acknowledged for statistical support. We also thank the patients and controls participating in this study. This research was supported as part of the St Göran bipolar project. This work was supported by grants from the Swedish foundation for Strategic Research, the Swedish Medical Research Council (K2011-61X -14647-09-3, K201061X-21569-01-1, and K2010-61P-21568-01-4), the Swedish Brain foundation, Märta Lundqvist's foundation, and the regional agreement on medical training and clinical research between Stockholm County Council and the Karolinska Institutet (ALF 20100305).

\section{REFERENCES}

Altshuler LL, Curran JG, Hauser P, Mintz J, Denicoff K, Post R (1995). T2 hyperintensities in bipolar disorder: magnetic resonance imaging comparison and literature meta-analysis. Am J Psychiatry 152: 1139-1144.

Andreazza AC, Cassini C, Rosa AR, Leite MC, de Almeida LM, Nardin P et al (2007). Serum S100B and antioxidant enzymes in bipolar patients. J Psychiatr Res 41: 523-529.

Belmaker RH (2004). Bipolar disorder. N Engl J Med 351: 476-486.

Beyer JL, Young R, Kuchibhatla M, Krishnan KR (2009). Hyperintense MRI lesions in bipolar disorder: a meta-analysis and review. Int Rev Psychiatry 21: 394-409.

Bjerke $M$, Andreasson U, Rolstad S, Nordlund A, Lind K, Zetterberg $\mathrm{H}$ et al (2009). Subcortical vascular dementia biomarker pattern in mild cognitive impairment. Dement Geriatr Cogn Disord 28: 348-356.

Bjerke $\mathrm{M}$, Zetterberg $\mathrm{H}$, Edman A, Blennow $\mathrm{K}$, Wallin $\mathrm{A}$, Andreasson U (2011). Cerebrospinal fluid matrix metalloproteinases and tissue inhibitor of metalloproteinases in combination with subcortical and cortical biomarkers in vascular dementia and Alzheimer's disease. J Alzheimers Dis 27: 665-676.

Bolandzadeh N, Davis JC, Tam R, Handy TC, Liu-Ambrose T (2012). The association between cognitive function and white matter lesion location in older adults: a systematic review. $B M C$ Neurol 12: 126.

Brettschneider J, Petzold A, Schottle D, Claus A, Riepe M, Tumani $\mathrm{H}$ (2006). The neurofilament heavy chain (NfH) in the cerebrospinal fluid diagnosis of Alzheimer's disease. Dement Geriatr Cogn Disord 21: 291-295.

Cha DS, McIntyre RS (2012). Treatment-emergent adverse events associated with atypical antipsychotics. Expert Opin Pharmacother 13: 1587-1598.

Chui HC (2007). Subcortical ischemic vascular dementia. Neurol Clin 25: 717-740.

Davis KA, Kwon A, Cardenas VA, Deicken RF (2004). Decreased cortical gray and cerebral white matter in male patients with familial bipolar I disorder. J Affect Disord 82: 475-485.

Ekman CJ, Lind J, Ryden E, Ingvar M, Landen M (2010). Manic episodes are associated with grey matter volume reduction - a voxel-based morphometry brain analysis. Acta Psychiatr Scand 122: 507-515.

Ekman M, Granstrom O, Omerov S, Jacob J, Landen M (2013). The societal cost of bipolar disorder in Sweden. Soc Psychiatry Psychiatr Epidemiol 48: 1601-1610.

Gunnarsson M, Malmestrom C, Axelsson M, Sundstrom P, Dahle $\mathrm{C}$, Vrethem $\mathrm{M}$ et al (2011). Axonal damage in relapsing multiple sclerosis is markedly reduced by natalizumab. Ann Neurol 69: 83-89.

Ho BC, Andreasen NC, Ziebell S, Pierson R, Magnotta V (2011). Long-term antipsychotic treatment and brain volumes: a longitudinal study of first-episode schizophrenia. Arch Gen Psychiatry 68: 128-137. 
Jakobsson J, Zetterberg H, Blennow K, Ekman CJ, Johansson AG, Landen M (2013). Altered concentrations of amyloid precursor protein metabolites in the cerebrospinal fluid of patients with bipolar disorder. Neuropsychopharmacology 38: 664-672.

Kapoor R, Furby J, Hayton T, Smith KJ, Altmann DR, Brenner R et al (2010). Lamotrigine for neuroprotection in secondary progressive multiple sclerosis: a randomised, double-blind, placebocontrolled, parallel-group trial. Lancet Neurol 9: 681-688.

Kempton MJ, Geddes JR, Ettinger U, Williams SC, Grasby PM (2008). Meta-analysis, database, and meta-regression of 98 structural imaging studies in bipolar disorder. Arch Gen Psychiatry 65: 1017-1032.

Kieseppa T, van Erp TG, Haukka J, Partonen T, Cannon TD, Poutanen VP et al (2003). Reduced left hemispheric white matter volume in twins with bipolar I disorder. Biol Psychiatry 54: 896-905.

Konarski JZ, McIntyre RS, Kennedy SH, Rafi-Tari S, Soczynska JK, Ketter TA (2008). Volumetric neuroimaging investigations in mood disorders: bipolar disorder versus major depressive disorder. Bipolar Disord 10: 1-37.

Krishnan MS, O’Brien JT, Firbank MJ, Pantoni L, Carlucci G, Erkinjuntti T et al (2006). Relationship between periventricular and deep white matter lesions and depressive symptoms in older people. The LADIS Study. Int J Geriatr Psychiatry 21: 983-989.

Kupfer DJ (2005). The increasing medical burden in bipolar disorder. JAMA 293: 2528-2530.

Lu CH, Petzold A, Kalmar B, Dick J, Malaspina A, Greensmith L (2012). Plasma neurofilament heavy chain levels correlate to markers of late stage disease progression and treatment response in SOD1(G93A) mice that model ALS. PLoS One 7: e40998.

Machado-Vieira R, Lara DR, Portela LV, Goncalves CA, Soares JC, Kapczinski F et al (2002). Elevated serum S100B protein in drugfree bipolar patients during first manic episode: a pilot study. Eur Neuropsychopharmacol 12: 269-272.

Martinez-Aran A, Vieta E, Colom F, Reinares M, Benabarre A, Gasto C et al (2000). Cognitive dysfunctions in bipolar disorder: evidence of neuropsychological disturbances. Psychother Psychosom 69: 2-18.

Martinez-Aran A, Vieta E, Colom F, Torrent C, Reinares M, Goikolea JM et al (2005). Do cognitive complaints in euthymic bipolar patients reflect objective cognitive impairment? Psychother Psychosom 74: 295-302.

McDonald C, Zanelli J, Rabe-Hesketh S, Ellison-Wright I, Sham P, Kalidindi S et al (2004). Meta-analysis of magnetic resonance imaging brain morphometry studies in bipolar disorder. Biol Psychiatry 56: 411-417.

Merikangas KR, Jin R, He JP, Kessler RC, Lee S, Sampson NA et al (2011). Prevalence and correlates of bipolar spectrum disorder in the world mental health survey initiative. Arch Gen Psychiatry 68: $241-251$.

Minden SL, Schiffer RB (1990). Affective disorders in multiple sclerosis. Review and recommendations for clinical research. Arch Neurol 47: 98-104.

Moore GJ, Bebchuk JM, Wilds IB, Chen G, Manji HK (2000). Lithium-induced increase in human brain grey matter. Lancet 356: $1241-1242$.

Olsson B, Zetterberg H, Hampel H, Blennow K (2011). Biomarkerbased dissection of neurodegenerative diseases. Prog Neurobiol 95: 520-534.

Palsson E, Figueras C, Johansson AG, Ekman CJ, Hultman B, Ostlind $\mathrm{J}$ et al (2013). Neurocognitive function in bipolar disorder: a comparison between bipolar I and II disorder and matched controls. BMC Psychiatry 13: 165.

Pelsers MM, Hanhoff T, Van der Voort D, Arts B, Peters M, Ponds $\mathrm{R}$ et al (2004). Brain- and heart-type fatty acid-binding proteins in the brain: tissue distribution and clinical utility. Clin Chem 50: $1568-1575$.
Perlis RH (2011). Translating biomarkers to clinical practice. Mol Psychiatry 16: 1076-1087.

Perrot R, Berges R, Bocquet A, Eyer J (2008). Review of the multiple aspects of neurofilament functions, and their possible contribution to neurodegeneration. Mol Neurobiol 38: 27-65.

Rajkowska G, Miguel-Hidalgo JJ (2007). Gliogenesis and glial pathology in depression. CNS Neurol Disord Drug Targets 6: 219-233.

Robinson LJ, Thompson JM, Gallagher P, Goswami U, Young AH, Ferrier IN et al (2006). A meta-analysis of cognitive deficits in euthymic patients with bipolar disorder. J Affect Disord 93: 105-115.

Rosengren LE, Karlsson JE, Karlsson JO, Persson LI, Wikkelso C (1996). Patients with amyotrophic lateral sclerosis and other neurodegenerative diseases have increased levels of neurofilament protein in CSF. J Neurochem 67: 2013-2018.

Rosso IM, Killgore WD, Cintron CM, Gruber SA, Tohen M, Yurgelun-Todd DA (2007). Reduced amygdala volumes in firstepisode bipolar disorder and correlation with cerebral white matter. Biol Psychiatry 61: 743-749.

Ryden E, Thase ME, Straht D, Aberg-Wistedt A, Bejerot S, Landen M (2009). A history of childhood attention-deficit hyperactivity disorder (ADHD) impacts clinical outcome in adult bipolar patients regardless of current ADHD. Acta Psychiatr Scand 120: 239-246.

Salzer J, Svenningsson A, Sundstrom P (2010). Neurofilament light as a prognostic marker in multiple sclerosis. Mult Scler 16: 287-292.

Sanchez-Moreno J, Martinez-Aran A, Colom F, Scott J, TabaresSeisdedos R, Sugranyes G et al (2009). Neurocognitive dysfunctions in euthymic bipolar patients with and without prior history of alcohol use. J Clin Psychiatry 70: 1120-1127.

Schroeter ML, Abdul-Khaliq H, Diefenbacher A, Blasig IE (2002). S100B is increased in mood disorders and may be reduced by antidepressive treatment. Neuroreport 13: 1675-1678.

Schroeter ML, Abdul-Khaliq H, Krebs M, Diefenbacher A, Blasig IE (2008). Serum markers support disease-specific glial pathology in major depression. J Affect Disord 111: 271-280.

Schroeter ML, Sacher J, Steiner J, Schoenknecht P, Mueller K (2013). Serum S100B represents a new biomarker for mood disorders. Curr Drug Targets 14: 1237-1248.

Sjogren M, Rosengren L, Minthon L, Davidsson P, Blennow K, Wallin A (2000). Cytoskeleton proteins in CSF distinguish frontotemporal dementia from AD. Neurology 54: 1960-1964.

Strakowski SM, Wilson DR, Tohen M, Woods BT, Douglass AW, Stoll AL (1993). Structural brain abnormalities in first-episode mania. Biol Psychiatry 33: 602-609.

Whitaker JN, Lisak RP, Bashir RM, Fitch OH, Seyer JM, Krance R et al (1980). Immunoreactive myelin basic protein in the cerebrospinal fluid in neurological disorders. Ann Neurol 7: 58-64.

Yatham LN, Kennedy SH, Schaffer A, Parikh SV, Beaulieu S, O'Donovan C et al (2009). Canadian Network for Mood and Anxiety Treatments (CANMAT) and International Society for Bipolar Disorders (ISBD) collaborative update of CANMAT guidelines for the management of patients with bipolar disorder: update 2009. Bipolar Disord 11: 225-255.

Zarate CA Jr, Tohen M, Land M, Cavanagh S (2000). Functional impairment and cognition in bipolar disorder. Psychiatr Q 71: 309-329.

Zetterberg H, Jacobsson J, Rosengren L, Blennow K, Andersen PM (2007). Cerebrospinal fluid neurofilament light levels in amyotrophic lateral sclerosis: impact of SOD1 genotype. Eur J Neurol 14: 1329-1333.

Supplementary Information accompanies the paper on the Neuropsychopharmacology website (http://www.nature.com/npp) 